\title{
Finite Element Approximation of Solutions to a Class of Nonlinear Hyperbolic-Parabolic Equations
}

\author{
Donald A. French* \\ Department of Mathematical Sciences, University of Cincinnati, Cincinnati, OH 45221 \\ Søren Jensen ${ }^{\dagger}$ \\ Department of Mathematics and Statistics, University of Maryland Baltimore County, Baltimore, MD 21250 \\ Thomas I. Seidman ${ }^{\ddagger}$ \\ Department of Mathematics and Statistics, University of Maryland Baltimore County, Baltimore, MD 21250 \\ January 13, 1999

\begin{abstract}
The numerical approximation of Antman and Seidman's model [AS] of the longitudinal motion of a viscoelastic rod is investigated. Their constitutive assumptions ensure that infinite compressive stress is needed to produce total compression of the rod. Analyses of the regularity of the solution of the continuous problem, the convergence of a semi-discrete finite element method, and the properties of a space-time finite element scheme are furnished. Results of a sample computation are also provided.
\end{abstract}

\section{Introduction:}

Consider the following model for the longitudinal motion of a visco-elastic rod:

$$
w_{t t}=\left[n\left(w_{s}, w_{s t}\right)\right]_{s}+f
$$

where $w=w(s, t)$ is the position at time $t$ of the point with reference position $s$ so $w_{s}$ is the strain and $w_{s t}$ is the strain rate; $n$ is then the contact force and $f$ is the body force. A natural set

\footnotetext{
${ }^{*}$ Work was carried out while French was a visitor at the University of Maryland Baltimore County during 1995-6, at his current position at the University of Cincinnati during 1996-7, and while he was on sabbatical at the Institute for Mathematics and its Applications and the University of Minnesota Mathematics Department during 1997-8. His research was supported in part by the Taft Foundation at the University of Cincinnati through their Grants-in-Aid program.

${ }^{\dagger}$ Research partially supported by the ONR Contract No. N00014-90-J-1238.

${ }^{\ddagger}$ Research partially supported by grants AFOSR-82-0271 and AFOSR-87-0190
} 
of boundary conditions for this problem consists of the specification of the contact forces at the endpoints;

$$
\left.n\left(w_{s}, w_{s t}\right)\right|_{s=0}=\left.n_{0}(t) \quad n\left(w_{s}, w_{s t}\right)\right|_{s=1}=n_{1}(t)
$$

where $n_{0}$ and $n_{1}$ are given. The initial conditions we use are

$$
w(\cdot, 0)=w_{0} \quad \text { and } \quad w_{t}(\cdot, 0)=v_{0}
$$

where $w_{0}$ and $v_{0}$ are given. For simplicity we have restricted our attention to a homogeneous rod with constant mass density equal to 1 and the constitutive function $n$ independent of $s$.

This model was considered in [AS] under assumptions on the constitutive function $n$ permitting fully nonlinear dependence on the strain rate while ensuring well-posedness and that one never develops 'infinite compression': that is, $w_{s}$ is pointwise bounded away from 0 .

Observe that in terms of the function $w$ the equation (1) is essentially hyperbolic. However, if we reformulate it in terms of $u:=w_{s}$ and $v:=w_{t}$ to obtain

$$
u_{t}=v_{s} \quad \text { and } \quad v_{t}=\left[n\left(u, v_{s}\right)\right]_{s}+f
$$

the second equation is parabolic in $v$ if we think of $u$ as being known.

It will also be convenient to split the contact force:

$$
n(y, z)=\phi^{\prime}(y)+\sigma(y, z)
$$

so $\phi$ is the elastic potential and $\sigma(y, z)$ is the viscous part. Here we will impose the following set of constitutive hypotheses: (much as in 'Note Added in Proof' of [AS])

(H0) (Properties of $\phi$ ) The elastic potential $\phi(y)$ is minimized at $y=1$ (equilibrium) so that $\phi:(0, \infty) \rightarrow[0, \infty)$ and one has $\phi^{\prime}(y) \rightarrow \infty$ as $y \rightarrow 0, \infty$.

(H1) (uniform ellipticity) There is some $m>0$ such that

$$
n_{z}(y, z) \geq m \quad \text { for } z \in \mathbb{R}, y>0,
$$

and thus $\sigma(y, z) z \geq m z^{2}$.

(H2)(Control of compression) There are $A, M>0$ and a (nonincreasing) function $\psi:\left(0, y_{*}\right) \rightarrow$ $(0, \infty)$ with $\psi(y) \rightarrow \infty$ as $y \rightarrow 0+$ such that, for some $y_{*}>0$

$$
n(y, z) \leq A+M \psi(y)-\psi^{\prime}(y) z
$$

for $z \in \mathbb{R}, 0<y<y_{*}$.

(H3) (Growth of $n$ ) For each $\eta>0$ there exists $\lambda=\lambda(\eta)$ such that

$$
\left|n_{y}(y, z)\right| \leq \lambda n_{z}(y, z) \quad \text { and } \quad\left|n_{y}(y, z)\right| \leq \lambda \sqrt{n_{z}(y, z)} \sqrt{\frac{|\sigma(y, z)|}{|z|}}
$$


for $z \in \mathbb{R}, y>\eta$.

In this report we provide a survey of our initial investigations into numerical methods for this highly nonlinear problem. In section 2 an analysis of the solution to the continuous problem, (1)(3) is given. We show several energy estimates and demonstrate the lower bound estimate for the strain $w_{s}$. In section 3 an example is given by explicit formula for a contact force function $n$ which satisfies hypotheses (H0)-(H3) (as well as (H4) and (H5) which will be introduced later). In sections 4 and 5 a semi-discrete finite element is introduced (discrete in the space variable $s$ and continuous in time $t$ ), energy estimates and the strain lower bound property are derived for the approximation function $w^{h}$ and an error estimate is provided. In section 6 an implicit fully discrete numerical method is used in a sample computation. This scheme uses the finite elements from section 4 and a simple centered difference scheme for the time discretization. Finally, in section 7 another fully discrete method is described using finite elements in both space and time. The special feature of this third method is that it preserves the lower bound property for the numerical strain, $w_{s}^{h k}$.

We will use $C$ generically to denote a positive constant for which we have an upper bound either absolute or depending only on the various parameters in (H0)-(H3), on the data norms, on $T$, and on previous occurrences of $C$, but not dependent on the particular data or the particular solutions involved. In particular, $C$ will never be dependent on the mesh parameter $h$ which we will introduce in section 4.

We note that this paper is a continuation of the work by the first two authors in [FJ]. In that paper energy properties of a space-time finite element method which resembled those for a certain viscoelastic rod problem were derived. In that model, however, the possibility of total compression was not considered as it is in this work.

Acknowledgement: The second author, Søren Jensen, passed away during the course of this work. He is greatly missed by us all. We acknowledge his significant contributions and participation in all aspects of this project except the final writing.

\section{The Continuous Problem:}

In this section we will provide a simplified version of the principal results of [AS], restricted to the context described above. The principal effort is to obtain a priori estimates for the solution, in particular obtaining pointwise estimates for the arguments $w_{s}$ and $w_{s t}$. We fix $T>0$ arbitrarily and assume the following, in addition to the constitutive hypotheses (H0)-(H3) above:

(A0) The data for the problem, functions $f, n_{0}, n_{1}, w_{0}$ and $v_{0}$ are as smooth as needed. Also $w_{0, s} \geq y_{*}>0$ with $y_{*}$ as in (H2).

\section{First energy estimate:}

The total energy for the rod (kinetic plus elastic potential) is

$$
E(t)=\frac{1}{2}\left\|w_{t}\right\|^{2}+\int_{0}^{1} \phi\left(w_{s}\right) d s
$$


where $\|\cdot\|$ is the $L^{2}(0,1)$ norm $\left(\|\cdot\|_{\infty}\right.$ will denote the $L^{\infty}(0,1)$ norm) and the work done by dissipated stress is

$$
W(t):=\int_{0}^{t}\left[\int_{0}^{1} \sigma\left(w_{s}, w_{s t}\right) w_{s t} d s\right] d t
$$

Using the equation (1) and an integration by parts gives

$$
\begin{aligned}
\frac{d}{d t}[E+W] & =\int_{0}^{1} f w_{t} d s+\left.n_{1} w_{t}\right|_{s=1}-\left.n_{0} w_{t}\right|_{s=0} \\
& \leq \frac{1}{2}\|f\|^{2}+\frac{1}{2}\left\|w_{t}\right\|^{2}+\frac{1}{2}\left|n_{1}\right|^{2}+\frac{1}{2}\left|n_{0}\right|^{2}+\epsilon \int_{0}^{1} \sigma\left(w_{s}, w_{s t}\right) w_{s t} d s+C_{\epsilon}\left\|w_{t}\right\|^{2}
\end{aligned}
$$

where we used the fact that for any $\epsilon>0$ there is a constant $C_{\epsilon}$ such that

$$
|g(s)| \leq \epsilon\left\|g_{s}\right\|+C_{\epsilon}\|g\|
$$

for any $s \in(0,1)$ and any $g \in H^{1}$. In view of (H1),

$$
\left\|w_{s t}\right\| \leq C\left[\left\|w_{t}\right\|^{2}+2 \int_{0}^{1} \sigma\left(w_{s}, w_{s t}\right) w_{s t} d s\right]^{1 / 2} .
$$

Taking $\epsilon=\frac{1}{2}$ to subtract that term from $d W / d t$, integrating over $(0, t)$, and applying the Gronwall inequality, we obtain the estimates:

$$
\left\|w_{t}(\cdot, t)\right\|, \Phi(t):=\int_{0}^{1} \phi\left(w_{s}\right) d s, W(t) \leq C
$$

\section{Lower bound for $w_{s}$ :}

Suppose one has $w_{s}(\bar{s}, \bar{t})<y_{*}$ for some $(\bar{s}, \bar{t}) \in \mathcal{Q}:=(0,1) \times[0, T]$. Then there is a $\tau>0$ such that $w_{s}(\bar{s}, t)<w_{s}(\bar{s}, \tau)=y_{*}$ for $\tau<t \leq \bar{t}$. Integrating (H2) with $y=w_{s}, z=w_{s t}$ over $(\tau, t)$ then gives

$$
\psi\left(w_{s}(\bar{s}, t)\right)-\psi\left(y_{*}\right)=\int_{\tau}^{t} \psi^{\prime}\left(w_{s}\right) w_{s t} d \hat{t} \leq \int_{\tau}^{t}\left[A+M \psi\left(w_{s}\right)-n\left(w_{s}, w_{s t}\right)\right] d \hat{t} .
$$

Since integrating (4) over $(0, \bar{s})$ gives $\left.n\left(w_{s}, w_{s t}\right)\right|_{\bar{s}, t}=n_{0}+\int_{0}^{\bar{s}}\left[w_{t t}-f\right] d s$, we then have

$$
\psi\left(w_{s}(\bar{s}, t)\right) \leq\left[\psi\left(y_{*}\right)+A(t-\tau)-\int_{\tau}^{t} n_{0} d \hat{t}+\left.\left(\int_{0}^{\bar{s}} w_{t} d s\right)\right|_{\tau} ^{\bar{t}}-\int_{\tau}^{t} \int_{0}^{\bar{s}} f d s d \hat{t}\right]+M \int_{\tau}^{t} \psi\left(\left.w_{s}\right|_{\bar{s}}\right) d \hat{t}
$$

The bracketed terms on the right can all be estimated (using (9) for the $\int w_{t t} d s$ terms) so the Gronwall inequality gives an upper bound for $\psi\left(w_{s}(\bar{s}, \bar{t})\right)$, which we note is uniform for any $(\bar{s}, \bar{t}) \in$ 
$(\mathcal{Q})$ for which $w_{s}(\bar{s}, \bar{t})<y_{*}$. Since $\psi(y) \rightarrow \infty$ as $y \rightarrow 0$, this implies a positive pointwise lower bound:

$$
w_{s}(s, t) \geq \bar{y}_{*}>0 \text {. }
$$

\section{Second energy estimate:}

The equation (1), formally differentiated with respect to $t$, gives

$$
w_{t t t}=\left[n_{y} w_{s t}+n_{z} w_{s t t}\right]_{s}+f_{t} .
$$

Using $2 w_{t t}$ as test function in the weak form of this gives

$$
\begin{aligned}
\left\|w_{t t}\right\|^{2}+2 \int_{0}^{t} \int_{0}^{1} n_{z} w_{s t t}^{2} d s d \hat{t}= & \left\|w_{t t}(\cdot, 0)\right\|^{2}+2\left(\left.\int_{0}^{t} n_{1} w_{t t}\right|_{s=1} d \hat{t}+\left.\int_{0}^{t} n_{0} w_{t t}\right|_{s=0} d \hat{t}\right) \\
& +\int_{0}^{t} \int_{0}^{1}\left(f_{t} w_{t t}-n_{y} w_{s t} w_{s t t}\right) d s d t \\
\leq & \left\|w_{t t}(\cdot, 0)\right\|^{2}+\int_{0}^{t} a\left\|w_{s t t}\right\| d \hat{t}+2 \lambda \int_{0}^{t} \int_{0}^{1}\left|\sqrt{n_{z}} w_{s t}\right| \sqrt{\sigma w_{s t t}} d s d \hat{t}
\end{aligned}
$$

where $a \in L^{2}(0, T)$ depends on the data $f, n_{0}, n_{1}$. This gives

$$
\int_{0}^{t} a\left\|w_{s t t}\right\| \leq\|a\|^{2}+\epsilon \int_{0}^{t} \int_{0}^{1} n_{z} w_{s t t}^{2} d \hat{t}+C_{\epsilon} \int_{0}^{t}\left\|w_{t t}\right\| d \hat{t},
$$

and we have estimated

$$
\left|n_{y} w_{s t} w_{s t t}\right| \leq \lambda\left|\sqrt{n_{z}} w_{s t t}\right| \sqrt{\left|\sigma w_{s t}\right|} \leq \epsilon n_{z} w_{s t t}^{2}+C_{\epsilon}\left|\sigma w_{s t}\right|
$$

using (8) with $y=w_{s}, z=w_{s t}$. It follows that

$$
\left\|w_{t t}\right\|^{2}+\int_{0}^{t} \int_{0}^{1} n_{z} w_{s t t}^{2} d s d \hat{t} \leq\left[\left\|w_{t t}(\cdot, 0)\right\|^{2}+\|a\|^{2}+C_{\epsilon} \int_{0}^{t} \int_{0}^{1} \sigma w_{s t} d s d \hat{t}\right]+C_{\epsilon} \int_{0}^{t}\left\|w_{t t}\right\|^{2} .
$$

Noting that the double integral term on the right side of the equation is just $W(t)$, for which we already have the bound (9), we apply the Gronwall inequality and obtain the estimates:

$$
\left\|w_{t t}\right\|, \quad \int_{0}^{t}\left\|w_{s t t}\right\|^{2} d \hat{t} \quad \leq C
$$

\section{Third energy estimate:}

If we rewrite (4) as $w_{t t}=\left[n_{y} w_{s s}+n_{z} w_{s s t}\right]+f$ we can solve for $w_{s s t}$

$$
w_{s s t}=\frac{w_{t t}-f}{n_{z}}-\left(\frac{n_{y}}{n_{z}}\right) w_{s s} .
$$


Taking norms and using (H1), (H3), gives

$$
\left\|w_{s s t}\right\| \leq C\left[\left\|w_{t t}\right\|+\frac{1}{m}\|f\|\right]+\lambda\left\|w_{s s}\right\|
$$

and, integrating,

$$
\left\|w_{s s}\right\| \leq\left\|w_{s s}(\cdot, 0)\right\|+\frac{1}{m} \int_{0}^{t}\left[\left\|w_{t t}\right\|+\|f\|\right] d \hat{t}+\lambda \int_{0}^{t}\left\|w_{s s}\right\| d \hat{t} .
$$

Applying the Gronwall inequality to this bounds $\left\|w_{s s}(\cdot, t)\right\|$ and using that in (13) gives:

$$
\left\|w_{s s}\right\|, \quad\left\|w_{s s t}\right\| \leq C
$$

Pointwise bounds for $w_{s}$ and $w_{s t}$ :

To obtain pointwise bounds for $w_{s}$ and $w_{s t}$ we need (14) and knowledge that $w_{s}$ and $w_{s t}$ are bounded at some point $s$ on $[0,1]$ to apply the Poincaré inequality: in particular at $s=0$ and $s=1$.

Observe from (H1) that $n$ is invertible in its second argument. Thus there is a function $g=$ $g(u, n)$ such that $g(y, n(y, z))=z$. Further, from (H1) and (H3) we have,

$$
g_{u}=-\frac{n_{y}}{n_{z}} \quad \text { so } \quad\left|g_{u}\right| \leq \lambda
$$

and

$$
g_{n}=\frac{1}{n_{z}} \quad \text { so } \quad\left|g_{n}\right| \leq \frac{1}{m} .
$$

Since $n=n_{0}$ at $s=0$ and $n=n_{1}$ at $s=1$ we have that $n$ is a $C^{1}$ function at $s=0$ or $s=1$. The new definition of the function $g$ allows us to specify $w_{s}$ by an ordinary differential equation (ODE) which is

$$
\frac{d}{d t} w_{s}=g\left(w_{s}, n\right)
$$

where as noted above $n$ is specified as a function of $t$ at $s=0$ and $s=1$. Combining the results on the bounds for $g$ and the defining ODE we conclude that both $w_{s}$ and $w_{s t}$ are bounded at $s=0$ and $s=1$ as asserted. These facts combined with (14) and (10) allow us to conclude that

$$
0<\bar{y}_{*} \leq w_{s} \leq \bar{y}^{*} \text { and }\left|w_{s t}\right| \leq \bar{z}^{*}
$$

where both results are on the space time domain $\mathcal{Q}$. ¿From now on, keeping (15) in mind, we will assume pointwise uniform bounds on $n, n_{y}, n_{z}, n_{z y}$ and $n_{z z}$.

The above results and arguments can be easily used to prove there exists a unique solution to the problem. We refer the reader to $[\mathrm{AS}]$ for more on these technical aspects. We are now in a position to state our main theorem on the continuous problem. 
Theorem 1: (Global existence and uniqueness): Suppose the data for the problem satisfy assumption (A0) and the constitutive function $n$ satisfies the hypotheses (H0)-(H3). Then the initial/boundary value problem defined by (1)-(3) has a unique solution, $w$, on the domain $\mathcal{Q}$ for any $T>0$ and $w$ satisfies the uniform norm inequalities (9), (12), (14), and (15).

\section{Example Constitutive Law:}

To show the consistency of the set of hypotheses (H0)-(H3) (as well as (H4) and (H5) which will be introduced later) we provide an explicit formula for $n(y, z)$ which satisfies them. It is this constitutive law which will be used later for the computational example. The functions $n$ and $n_{z}$ will be continuous and the derivatives $n_{y}, n_{y z}$ and $n_{z z}$ will be defined on the domain of definition which is $y>0$ and $-\infty<z<\infty$.

We take

$$
\phi(y)=\frac{2}{y}+y^{2}
$$

to satisfy the hypothesis (H0). The definition of $\sigma$ is much more complicated;

$$
\sigma(y, z)= \begin{cases}z-\frac{1}{2} z^{2} & \text { if } z \leq 0 \text { and } y \geq 1 \\ z-\frac{1}{2} z^{2}-\frac{1}{2}\left(1-y^{-2}\right)^{2} & \text { if } z \leq 0 \text { and }(1-z)^{-1 / 2} \leq y<1 \\ z y^{-2} & \text { if } z \leq 0 \text { and } 0<y<(1-z)^{-1 / 2} \\ z & \text { if } z>0 \text { and } y \geq 1 \\ z+\left(y^{-2}-1\right) \beta(z) & \text { if } z>0 \text { and } 0<y<1\end{cases}
$$

where

$$
\beta(z)= \begin{cases}z+z^{2}-z^{3} & \text { if } 0 \leq z \leq 1 \\ 1 & \text { if } z>1\end{cases}
$$

Note that $\sigma(y, z) / z \geq 1$; this will be used later to verify the second part of (H3). To check hypothesis (H1) we differentiate $\sigma$ with respect to $z$. This gives

$$
\sigma_{z}(y, z)= \begin{cases}1-z & \text { if } z \leq 0 \text { and } y \geq(1-z)^{-1 / 2} \\ y^{-2} & \text { if } z \leq 0 \text { and } 0<y<(1-z)^{-1 / 2} \\ 1 & \text { if } z>0 \text { and } y \geq 1 \\ 1+\left(y^{-2}-1\right) \beta^{\prime}(z) & \text { if } z>0 \text { and } 0<y<1\end{cases}
$$

where

$$
\beta^{\prime}(z)= \begin{cases}1+2 z-3 z^{2} & \text { if } 0 \leq z \leq 1 \\ 0 & \text { if } z>1\end{cases}
$$

It follows that (H1) holds with $m=1$; a crucial step is to note that $\beta^{\prime}(z) \geq 0$ for $0 \leq z \leq 1$. Also note here that $\sigma_{y z}$ and $\sigma_{z z}$ are defined and piecewise continuous. 
To verify (H2) we take $\psi(y)=y^{-1}$ and $y_{*}=1$. It follows that $\psi^{\prime}(y)=-y^{-2}$ and thus the upper bound on $n$ is

$$
A+M y^{-1}+z y^{-2}
$$

in the region where $0<y \leq 1$. Perhaps the most difficult verification is in the region where $(1-z)^{-1 / 2} \leq y \leq 1$ and $z \leq 0$. Here, we have

$$
n(y, z)=2\left(-y^{-2}+y\right)+\left(z-\frac{1}{2} z^{2}-\frac{1}{2}\left(1-y^{-2}\right)^{2}\right) \leq 1+z \leq 1+z y^{-2}
$$

which gives (H2) if we take $A \geq 1$ and $M \geq 2$.

To verify (H3) we will need to evaluate the $y$-derivative.

$$
n_{y}(y, z)=\left(-4 y^{-3}+2\right)+\sigma_{y}(y, z)
$$

with

$$
\sigma_{y}(y, z)= \begin{cases}0 & \text { if } z \leq 0 \text { and } y \geq 1 \\ 2\left(1-y^{-2}\right) y^{-3} & \text { if } z \leq 0 \text { and }(1-z)^{-1 / 2} \leq y<1 \\ -2 z y^{-3} & \text { if } z \leq 0 \text { and } 0<y<(1-z)^{-1 / 2} \\ 0 & \text { if } z>0 \text { and } y \geq 1 \\ -2 y^{-3} \beta(z) & \text { if } z>0 \text { and } 0<y<1 .\end{cases}
$$

Thus, in the region where $z \geq 0$ and $y \geq \eta>0$ we have

$$
\left|n_{y}(y, z)\right| \leq 6 y^{-3}+2 \leq\left(6 \eta^{-3}+2\right) \sqrt{\left|\sigma_{z}(y, z)\right|} \sqrt{\frac{\sigma(y, z)}{z}}=\left(6 \eta^{-3}+2\right) \sqrt{\left|n_{z}(y, z)\right|} \sqrt{\frac{\sigma(y, z)}{z}} .
$$

The other inequalities follow similarly in the other regions.

\section{The Semi-Discrete Finite Element Method:}

Let $V^{h}$ be the space of continuous piecewise linear functions defined on the partition with nodes $s_{p}=p h$ and parameter $h=P^{-1}$ where $P$ is a positive integer; we embed this in $L^{2}(0,1)$ with the induced inner product. Let $I_{p}=\left(s_{p-1}, s_{p}\right)$ and $w_{s}^{h, p}(t)=\left.w_{s}^{h}(\cdot, t)\right|_{I_{p}}$ which is a constant. A spatially discrete finite element method can then be constructed as follows: Find $w^{h}(\cdot, t) \in V^{h}$ for $0<t<T$ such that

$$
\left(w_{t t}^{h}, \chi\right)+\left(n\left(w_{s}^{h}, w_{s t}^{h}\right), \chi_{s}\right)=(f, \chi)+n_{1} \chi(1)-n_{0} \chi(0)
$$

for all $\chi \in V^{h}$ where $w^{h}(\cdot, 0) \cong w_{0}$ and $w_{t}^{h}(\cdot, 0) \cong v_{0}$. We observe that this problem has a unique solution, at least for short time. Since $V^{h}$ is a finite dimensional space, a basis for it can be found and from this a system of ordinary differential equations can be derived. As long as $n$ is well defined there exists at least a short time solution.

For the error estimate we will make the following regularity assumption: 
(A1) Function $w_{s t t} \in L^{\infty}(\mathcal{Q})$ and for all $t \in[0, T], w_{s s t t} \in L^{2}(0,1)$.

We also will need an extra smoothness assumption on the constitutive law, valid for our example. (H4) Functions $n_{y z}$ and $n_{z z}$ are piecewise continuous in $y, z$.

Note that our explicit constitutive formula (16)-(17) satisfies (H4). We also note that, without proof, an argument similar to those of section 2 (differentiate (11) yet again with respect to $t$, expand, and estimate the term on the right to get a differential inequality for $\left\|w_{t t t}\right\|^{2}$ ) gives (A1) for some time interval.

\section{Energy bounds for $w^{h}$ :}

One can apply the first energy argument of section 2 to the semi-discrete problem and show that, similarly,

$$
\int_{0}^{t}\left\|w_{s t}^{h}\right\|^{2} d \tau, \quad\left\|w_{t}\right\|, \quad \int_{0}^{1} \phi\left(w_{s}^{h}\right) d s \leq C .
$$

One can also apply the lower bound argument to obtain

$$
w_{s}^{h}(s, t) \geq \bar{y}_{*}^{S D}>0
$$

on $\mathcal{Q}$ where $\bar{y}_{*}^{S D}$ is a constant that is independent of $h$. The notation $S D$ stands for semi-discrete. The second energy argument can be applied by differentiating the variational equation defining $w^{h}$ with respect to $t$. This gives

$$
\left(w_{t t t}^{h}, \chi\right)+\left(n_{y} w_{s t}^{h}+n_{z} w_{s t t}^{h}, \chi_{s}\right)=\left(f_{t}, \chi\right)+n_{1}^{\prime} \chi(1)-n_{0}^{\prime} \chi(0) .
$$

By taking $\chi=2 w_{t t}^{h}$, integrating over time, one can show the same estimates for $w^{h}$ as was done in section 2 for $w$ :

$$
\left\|w_{t t}^{h}\right\|, \quad \int_{0}^{T}\left\|w_{t t s}^{h}\right\| d \tau \leq C
$$

\section{Bounds at the boundary:}

Define

$$
\chi_{p}(s)= \begin{cases}0 & \text { if } 0<s<s_{p-1} \\ s-s_{p-1} & \text { if } s_{p-1}<s<s_{p} \\ 1 & \text { if } s_{p}<s<1\end{cases}
$$

Then, taking $\chi_{P}$ as the test function in (18) gives the equation

$$
h n\left(w_{s}^{h, P}, w_{s t}^{h, P}\right)=\left(f, \chi_{P}\right)+h\left(n_{1}(t)-n_{0}(t)\right)-\left(w_{t t}^{h}, \chi_{P}\right) .
$$

Multiplying by $w_{s t}^{h, P}$ and noting that $\left\|\chi_{p}\right\|_{L^{\infty}(0,1)} \leq h$ we have

$$
m\left|w_{s t}^{h, P}\right| \leq\left(\|f\|_{L^{1}(0,1)}+\left|n_{1}(t)\right|+\left|n_{0}(t)\right|+\left\|w_{t t}^{h}\right\|\right)
$$


and, since the quantities on the right are bounded, we conclude that

$$
\left|w_{s t}^{h, P}\right| \leq C
$$

Since

$$
w_{s}^{h, P}(t)=w_{s}^{h, P}(0)+\int_{0}^{t} w_{s t}^{h, P}(\tau) d \tau
$$

we have that

$$
\left|w_{s}^{h, P}\right| \leq C
$$

A similar bound can be found for $w_{s}^{h, 0}(t)$.

Interior pointwise upper bound for $w_{s}^{h}$ :

Using $\chi=\chi_{p}$ in (18) we obtain

$$
h n\left(w_{s}^{h, p}, w_{s t}^{h, p}\right)=\left(f-w_{t t}^{h}, \chi_{p}\right)
$$

for each $0<p<P$. Subtracting the $(p-1)$ equation from the $p$ equation, we obtain

$$
h\left(n\left(w_{s}^{h, p}, w_{s t}^{h, p}\right)-n\left(w_{s}^{h, p-1}, w_{s t}^{h, p-1}\right)\right)=\left(f-w_{t t}^{h}, \chi_{p}-\chi_{p-1}\right)
$$

where, using the Taylor theorem along the segment joining $\left(w_{s}^{h, p-1}, w_{s t}^{h, p-1}\right)$ to $\left(w_{s}^{h, p}, w_{s t}^{h, p}\right)$,

$$
w_{s t}^{h, p}-w_{s t}^{h, p-1}=\frac{\left(f-w_{t t}^{h}, \chi_{p}-\chi_{p-1}\right)}{h n_{z}(\gamma, \zeta)}-\frac{n_{y}(\gamma, \zeta)}{n_{z}(\gamma, \zeta)}\left(w_{s}^{h, p}-w_{s}^{h, p-1}\right)
$$

for some pair $(\gamma, \zeta)$ on the segment. Define $\partial_{p} u^{h}=u^{h, p}-u^{h, p-1}$, where $u^{h}$ is any piecewise constant function defined on the mesh, as $w_{s}^{h}$ and $w_{s t}^{h}$. Then

$$
\sum_{p=1}^{P}\left|\partial_{p} w_{s t}^{h}\right| \leq \frac{1}{m} \sum_{p=1}^{P}\left\|f-w_{t t}\right\|_{L^{1}\left(I_{p}\right)}+\lambda \sum_{p=1}^{P}\left|\partial_{p} w_{s}^{h}\right|
$$

where $\lambda$ is defined in (H3). Since $\left\|f-w_{t t}^{h}\right\|_{L^{1}(0,1)}$ is bounded independently of $h$, it follows from a Gronwall argument that

$$
\sum_{p=1}^{P}\left|\partial_{p} w_{s}^{h}\right| \leq C
$$

and using this in (22) gives

$$
\sum_{p=1}^{P}\left|\partial_{p} w_{s t}^{h}\right| \leq C
$$


To obtain an upper bound on $w_{s}^{h, p}$ we have

$$
\begin{aligned}
w_{s}^{h, p} & =\partial_{p} w_{s}^{h}+w_{s}^{h, p-1} \\
& =\partial_{p} w_{s}^{h}+\partial_{p-1} w_{s}^{h}+\ldots \partial_{1} w_{s}^{h}+w_{s}^{h, 0}
\end{aligned}
$$

so

$$
\left|w_{s}^{h, p}\right| \leq \sum_{p=1}^{P}\left|\partial_{p} w_{s}^{h}\right|+\left|w_{s}^{h, 0}\right| \leq C .
$$

In the same way we as for (15) we also find

$$
\left|w_{s t}^{h, p}\right| \leq C
$$

We are now in a position to state our first theorem on the properties of the semi-discrete method.

Theorem 2 (Stability of the semi-discrete scheme): Assume the hypotheses (H0)-(H3) hold on $n$ and assumption (A0) is valid for the data. Then the numerical method defined by (18) has a unique solution which satisfies the inequalities (19), (20), and the pointwise bounds

$$
0<\bar{y}_{*}^{S D} \leq w_{s}^{h} \leq \bar{y}^{S D, *} \text { and }\left|w_{s t}^{h}\right| \leq \bar{z}^{S D, *} .
$$

As with the continuous solution we can assume pointwise bounds for $n, n_{y}, n_{z}, n_{z y}$, and $n_{z z}-$ uniform on the relevant domain.

A redefinition of $n(\cdot)$ off the estimated domain occurs in the existence proof but is not needed for implementation. It is important to realize that the implementation of this scheme does not involve any computation of the bounds (23) which we have noted to justify it.

\section{Error Estimate for the Semi-Discrete Method:}

In this section we will use the uniform bounds obtained in the previous section and a nonlinear Ritz projection to obtain an error estimate. We first define and analyze our projection, which is used in the error estimation but not in the actual computations.

Ritz Projection:

Consider finding $\nu^{h}(\cdot, t) \in V^{h}$ so for each $t$

$$
\left(n\left(w_{s}, \nu_{s}^{h}\right), \chi_{s}\right)=\left(n\left(w_{s}, w_{s t}\right), \chi_{s}\right)
$$

for all $\chi \in V^{h}$ where

$$
\int_{0}^{1} \nu^{h} d s=\int_{0}^{1} w_{t} d s
$$

We will now show that there exists a unique $\nu^{h}$ that satisfies (24) and (25) and view this construction as a nonlinear map, called the Ritz projection: $w_{t} \rightarrow \nu^{h}$. 


\section{Existence of the Ritz projection:}

We will use the following version of Brouwer's fixed-point theorem to show there exists a solution to (24) (see [GR], Corollary 1.1, p. 279):

Let $H$ be a finite-dimensional Hilbert space with inner product $\langle\cdot, \cdot\rangle$ and corresponding norm $|\cdot|$. Let $\Psi: H \rightarrow H$ be a continuous mapping with the following property. There exists a $\mu>0$ such that

$$
\langle\Psi(g), g\rangle \geq 0 \quad \forall g \in H \quad \text { with } \quad|g|=\mu .
$$

Then, there exists a $g^{*} \in H$ with $\left|g^{*}\right| \leq \mu$ such that $\Psi\left(g^{*}\right)=0$.

To apply this to our situation, we first rewrite (24) as follows: Instead of $\nu^{h}$ we seek $\tilde{\nu}^{h} \in V_{0}^{h}=$ $\left\{\chi \in V^{h}: \int_{0}^{1} \chi d s=0\right\}$ such that

$$
\left(n\left(w_{s}, \tilde{\nu}_{s}^{h}\right), \chi_{s}\right)=\left(n\left(w_{s}, w_{s t}\right), \chi_{s}\right)
$$

where $\tilde{\nu}^{h}=\nu^{h}-M_{v}$ with

$$
M_{v}=\int_{0}^{1} w_{t} d s=\int_{0}^{1} \nu^{h} d s .
$$

Note that this problem is equivalent to (24). We set the Hilbert space $H=V_{0}^{h}$ with the inner product

$$
\langle\xi, \zeta\rangle=\int_{0}^{1} \xi_{s} \zeta_{s} d s
$$

and take $g=\tilde{\nu}^{h}$. Then

$$
\langle\Psi(g), \zeta\rangle:=\left(n\left(w_{s}, g_{s}\right), \zeta_{s}\right)-\left(n\left(w_{s}, w_{s t}\right), \zeta_{s}\right)
$$

but

$$
\begin{aligned}
\langle\Psi(g), g\rangle & =\left(n\left(w_{s}, g_{s}\right), g_{s}\right)-\left(n\left(w_{s}, w_{s t}\right), g_{s}\right) \\
& \geq m\left\|g_{s}\right\|^{2}-\left\|n\left(w_{s}, w_{s t}\right)\right\|\left\|g_{s}\right\| \\
& =m\left(\mu^{2}-\frac{\alpha}{m} \mu\right)
\end{aligned}
$$

where $\alpha=\left\|n\left(w_{s}, w_{s t}\right)\right\|$ and $\mu=\left\|g_{s}\right\|$. For $\mu \geq \alpha / m$ the inequality (26) holds, implying there exists a solution to the projection problem (24).

\section{Uniqueness of the Ritz projection:}

Suppose (24) might have two solutions $\nu^{h}$ and $\bar{\nu}^{h}$. ¿From the defining equation (24) and Taylor's theorem we have

$$
\left(n_{z}\left(w_{s}, \xi\right)\left(\nu^{h}-\bar{\nu}^{h}\right)_{s}, \chi_{s}\right)=0
$$


for all $\chi \in V^{h}$ where $\xi$ lies between $\nu^{h}$ and $\bar{\nu}^{h}$. Choosing $\chi=\nu^{h}-\bar{\nu}^{h}$ and using hypothesis (H1) we have

$$
m\left\|\left(\nu^{h}-\bar{\nu}^{h}\right)_{s}\right\|^{2} \leq 0
$$

and, since also

$$
\int_{0}^{1}\left(\nu^{h}-\bar{\nu}^{h}\right) d s=0
$$

we must have $\nu^{h}=\bar{\nu}^{h}$.

\section{Error estimate for the Ritz projection:}

Let $b(z)=n\left(w_{s}, z\right)$. We will need the following approximation results. We assume there is an interpolation operator $\pi: H^{1}(0,1) \rightarrow V^{h}$ such that, for any $z \in H^{1}(0,1) \subset L^{\infty}(0,1)$,

$$
\|(I-\pi) z\| \leq C h\left\|z_{s}\right\| \text { and }\|\pi z\|_{L^{\infty}(0,1)} \leq\|z\|_{L^{\infty}(0,1)} .
$$

We will also use the following standard inverse inequality: for any $\chi \in V^{h}$

$$
\|\chi\|_{L^{\infty}\left(I_{p}\right)} \leq C h^{-1 / 2}\|\chi\|_{L^{2}\left(I_{p}\right)}
$$

where $I_{p}$ is an interval in the finite element mesh which has length $h$ (see $[\mathrm{C}]$ ). Let $\bar{b}$ be a $C^{1}$ function with $\bar{b}(z)=b(z)$ for $|z| \leq\left\|w_{s t}\right\|_{L^{\infty}(0,1)}+\delta$ where $\delta>0$ and such that

$$
\left\|\bar{b}^{\prime}\right\|_{L^{\infty}(0,1)} \leq R \text { and } \bar{b}^{\prime} \geq m \text {. }
$$

We will analyze the problem: find $\bar{\nu}^{h} \in V^{h}$ such that

$$
\left(\bar{b}\left(\bar{\nu}_{s}^{h}\right), \chi_{s}\right)=\left(\bar{b}\left(w_{s t}\right), \chi_{s}\right)
$$

for all $\chi \in V^{h}$ and will show that this gives

$$
\left\|\bar{\nu}_{s}^{h}-w_{s t}\right\| \leq C h\left\|w_{s s t}\right\| .
$$

¿From this we will have that

$$
\begin{aligned}
\left\|\bar{\nu}_{s}^{h}\right\|_{\infty} & \leq\left\|\bar{\nu}_{s}^{h}-\pi w_{s t}\right\|_{\infty}+\left\|\pi w_{s t}\right\|_{\infty} \\
& \leq C h^{-1 / 2}\left\|\bar{\nu}_{s}^{h}-\pi w_{s t}\right\|+\left\|w_{s t}\right\|_{\infty} \\
& \leq C h^{1 / 2}\left\|w_{s s t}\right\|+\left\|w_{s t}\right\|_{\infty}
\end{aligned}
$$

Choosing $h$ small enough so

$$
C h^{1 / 2}\left\|w_{s s t}\right\| \leq \delta
$$


implies that $\bar{b}=b$ for the range of $\bar{\nu}_{s}^{h}$ considered so, since we have uniqueness for (24), it now follows that $\bar{\nu}^{h}=\nu^{h}$ and, setting $\rho=\nu^{h}-w_{t}$,

$$
\left\|\rho_{s}\right\| \leq C h\left\|w_{s s t}\right\| \text {. }
$$

We now show (30). Note that

$$
\int_{0}^{1} \bar{b}^{\prime}\left(w_{s t}+\zeta \bar{\rho}_{s}\right) \bar{\rho}_{s} d \zeta=\bar{b}\left(\bar{\nu}^{h}\right)-\bar{b}\left(w_{s t}\right)
$$

where $\bar{\rho}=\bar{\nu}^{h}-w_{t}$. If we then let

$$
\bar{B}=\int_{0}^{1} \bar{b}^{\prime}\left(w_{s t}+\zeta \bar{\rho}_{s}\right) d \zeta
$$

then (29) becomes

$$
\left(\bar{B} \bar{\rho}_{s}, \chi_{s}\right)=0
$$

and $m \leq \bar{B} \leq M$. Then

$$
\left(\bar{B} \bar{\rho}_{s}, \bar{\rho}_{s}\right)=\left(\bar{B} \bar{\rho}_{s},(\pi-I) w_{s t}\right)
$$

SO

$$
m\left\|\bar{\rho}_{s}\right\| \leq M\left\|(I-\pi) w_{s t}\right\| \leq C h\left\|w_{s s t}\right\|
$$

proving (30).

Finally, we need an estimate on the $t$ derivative of the error. Differentiating the defining equation (24) gives

$$
\left(n_{z}\left(w_{s}, \nu_{s}^{h}\right) \nu_{s t}^{h}, \chi_{s}\right)+\left(n_{y}\left(w_{s}, \nu_{s}^{h}\right) w_{s t}, \chi_{s}\right)=\left(n_{z}\left(w_{s}, w_{s t}\right) w_{s t t}, \chi_{s}\right)+\left(n_{y}\left(w_{s}, w_{s t}\right) w_{s t}, \chi_{s}\right)
$$

or

$$
\left(n_{z}\left(w_{s}, \nu_{s}^{h}\right) \rho_{s t}, \chi_{s}\right)=-\left(\left(n_{z}\left(w_{s}, \nu_{s}^{h}\right)-n_{z}\left(w_{s}, w_{s t}\right)\right) w_{s t t}, \chi_{s}\right)-\left(\left(n_{y}\left(w_{s}, \nu_{s}^{h}\right)-n_{y}\left(w_{s}, w_{s t}\right)\right) w_{s t}, \chi_{s}\right) .
$$

Then, applying the Taylor theorem,

$$
\left(n_{z}\left(w_{s}, \nu_{s}^{h}\right) \rho_{s t}, \chi_{s}\right)=\left(D \rho_{s}, \chi_{s}\right)
$$

where

$$
D:=-\left(n_{z z}\left(w_{s}, \gamma\right) w_{s t t}+n_{y z}\left(w_{s}, \xi\right) w_{s t}\right) .
$$

By our assumptions we have that $D$ is bounded in $L^{\infty}$ and that $\gamma$ and $\xi$ each lie between $\nu_{s}^{h}$ and $w_{s t}$. Then

$$
\begin{aligned}
\left(n_{z} \rho_{s t}, \rho_{s t}\right) & =\left(n_{z} \rho_{s t}, \nu_{s t}^{h}\right)-\left(n_{z} \rho_{s t}, w_{s t t}\right) \\
& \left.=\left(D \rho_{s}, \nu_{s t}^{h}\right)-\left(n_{z} \rho_{s t}, w_{s t t}\right)+\left[-\left(D \rho_{s}, \pi w_{s t t}\right)+n_{z} \rho_{s t}, \pi w_{s t t}\right)\right] \\
& =\left(D \rho_{s}, \rho_{s t}\right)+\left(D \rho_{s},(I-\pi) w_{s t t}\right)+\left(n_{z} \rho_{s t},(I-\pi) w_{s t t}\right)
\end{aligned}
$$


or

$$
m\left\|\rho_{s t}\right\|^{2} \leq C\left(h\left\|\rho_{s t}\right\|+h^{2}\right)\left\|w_{s s t t}\right\|
$$

which implies

$$
\left\|\rho_{s t}\right\| \leq C h\left\|w_{s s t}\right\| .
$$

We are now in a position to state the key error estimate for the projection.

Lemma: Let $\nu^{h}$ be the Ritz projection of $w_{t}$ satisfying (24) and (25). Assume the conditions (H0)-(H4) hold for $n$, the data are smooth, (A0), and the solution $w$ is sufficiently regular, (A1). Then the error $\rho=\nu^{h}-w_{t}$ satisfies an estimate:

$$
\left\|\rho_{s}\right\| \leq C h\left\|w_{s s t}\right\| \text { and }\left\|\rho_{s t}\right\| \leq C h\left\|w_{s s t t}\right\| .
$$

\section{Error estimate for the semi-discrete problem:}

We start with a standard splitting for time dependent problems,

$$
e_{t}=w_{t}-w_{t}^{h}=\left(w_{t}-\nu^{h}\right)+\left(\nu^{h}-w_{t}^{h}\right)=\rho+\theta^{h},
$$

and then obtain

$$
\left(\theta_{t}^{h}, \chi\right)+\left(n\left(w_{s}, \nu_{s}^{h}\right)-n\left(w_{s}^{h}, w_{s t}^{h}\right), \chi_{s}\right)=-\left(\rho_{t}, \chi\right) .
$$

Choosing $\chi=\theta^{h}$ in (18) we obtain

$$
\begin{aligned}
\frac{1}{2} \frac{d}{d t}\left\|\theta^{h}\right\|^{2}+\left(n_{z}\left(w_{s}, \xi\right) \theta_{s}^{h}, \theta_{s}^{h}\right) & =-\left(\rho_{t}, \theta^{h}\right)-\left(n_{y}\left(\gamma, w_{s t}^{h}\right) e_{s}, \theta_{s}^{h}\right) \\
& =-\left(\rho_{t}, \theta^{h}\right)-\left(n_{y}\left(\gamma, w_{s t}^{h}\right) \int_{0}^{t}\left(\rho_{s}+\theta_{s}^{h}\right) d \tau, \theta_{s}^{h}\right)
\end{aligned}
$$

where $\gamma$ is between $w_{s}$ and $w_{s}^{h}$ and $\xi$ is between $w_{s t}$ and $w_{s t}^{h}$. Then

$$
\frac{d}{d t}\left\|\theta^{h}\right\|^{2}+\left\|\theta_{s}^{h}\right\|^{2} \leq C\left(\left\|\rho_{t}\right\|^{2}+\int_{0}^{t}\left(\left\|\rho_{s}\right\|^{2}+\left\|\theta_{s}^{h}\right\|^{2}\right) d \tau .\right.
$$

Apply the Gronwall inequality - assuming, for simplicity, that $w_{t}^{h}(\cdot, 0)=\nu^{h}(\cdot, t)$. This gives

$$
\left\|\theta^{h}\right\|^{2}+\int_{0}^{t}\left\|\theta_{s}^{h}\right\|^{2} d \tau \leq C h^{2}
$$

which, together with the estimates on $\rho$, gives the error estimate described in the theorem below. Theorem 3 (Semi-discrete error estimate): Suppose $w^{h}$ is the solution of (18) and $w$ is the solution of (1)-(3). Assume the conditions (H0)-(H4) hold for $n$; the data are smooth, (A0); the solution $w$ is sufficiently smooth as in $(\mathrm{A} 1)$, and $w^{h}(\cdot, 0)$ is the nonlinear Ritz projection of $w_{t}(\cdot, 0)$. Then

$$
\left\|w_{s t}-w_{s t}^{h}\right\|_{L^{2}(\mathcal{Q})} \leq C h .
$$




\section{Discretization in Time of the Finite Element Method:}

In this section we describe a simple fully discrete numerical method. We use the piecewise linear finite elements described previously for the spatial variable and introduce a centered finite difference procedure for the time discretization. Because of the parabolic nature of this problem with respect to $w_{t}$ we have chosen to make the scheme implicit. Partition $[0, T]$ into $Q$ intervals of length $k=T / Q$ with nodes $t_{q}=q k$. Let $J_{q}=\left(t_{q-1}, t_{q}\right)$ and $J=[0, T]$. We linearize the function $n$ with respect to the $z$ variable using Taylor's theorem about $z_{0}$,

$$
n(\cdot, z)=n\left(\cdot, z_{0}\right)+n_{z}\left(\cdot, z_{0}\right)\left(z-z_{0}\right) .
$$

Taking

$$
z=\frac{w_{s}^{h, q+1}-w_{s}^{h, q-1}}{2 k} \text { and } z_{0}=\frac{w_{s}^{h, q}-w_{s}^{h, q-1}}{k}
$$

leads to the finite element/finite difference method:

$$
\begin{aligned}
\left(w_{s}^{h, q+1}-2 w_{s}^{h, q}+w_{s}^{h, q-1}, \chi\right)+ & k^{2}\left[\left(\left(n\left(w_{s}^{h, q}, \frac{w_{s}^{h, q}-w_{s}^{h, q-1}}{k}\right), \chi_{s}\right)\right.\right. \\
& \left.+\left(n_{z}\left(w_{s}^{h, q}, \frac{w_{s}^{h, q}-w_{s}^{h, q-1}}{k}\right) \frac{w_{s}^{h, q+1}-2 w_{s}^{h, q}+w_{s}^{h, q-1}}{2 k}, \chi_{s}\right)\right] \\
= & k^{2}\left((f, \chi)+n_{1} \chi(1)-n_{0} \chi(0)\right) .
\end{aligned}
$$

Specifying basis functions $\left\{\chi_{0}, \ldots, \chi_{P}\right\}$, we then obtain a system of equations of the form

$$
\left(M+k K^{q}\right) W^{q+1}=F^{n}
$$

where $M$ is the mass matrix, $K^{q}$ is a stiffness matrix, $W^{q}$ has the coefficients of the basis functions in $w^{h, q}$, and $F^{q}$ has all the other terms that involve quantities on the $q$ and $q-1$ time levels. Note that

$$
M_{i j}=\left(\chi_{i}, \chi_{j}\right), \quad K_{i j}^{q}=\left(n_{z}\left(w_{s}^{h, q}, \frac{w_{s}^{h, q}-w_{s}^{h, q-1}}{k}\right) \chi_{j, s}, \chi_{i, s}\right) \text { and } w^{h, q}(s)=\sum_{j=0}^{P} W_{j}^{q} \chi_{j}(s) .
$$

As starting data for this multistep method we take

$$
w^{h, 0}\left(s_{p}\right) \cong w\left(s_{p}, 0\right) \text { and } w^{h, 1}\left(s_{p}\right) \cong w\left(s_{p}, 0\right)+k w_{t}\left(s_{p}, 0\right)
$$

for $p=0,1, \ldots, P$. The trapezoid rule is used for the evaluation of all the spatial integrals that arise. 
The accuracy of the method is of interest. To examine this we created a simple test example with a known solution

$$
w(s, t)=e^{0.2 s}(2-\sin t),
$$

noting that $w_{s}(s, t) \geq 0.2$ for $0<s<1$ and $t>0$. For the data for this problem we let

$$
f=w_{t t}-n_{y}\left(w_{s}, w_{s t}\right) w_{s s}-n_{z}\left(w_{s}, w_{s t}\right) w_{s s t}, \quad n_{0}(t)=n\left(w_{s}(0, t), w_{s t}(0, t)\right),
$$

and

$$
n_{1}(t)=n\left(w_{s}(1, t), w_{s t}(1, t)\right)
$$

where all quantities involving $w$ are defined using (34). We then did several test runs on this problem and found that the observed convergence rate for the $L^{\infty}$ norm of the difference of $w(\cdot, 1)$ and $w^{h, 1 / k}$ was close to $O\left(h^{2}+k^{2}\right)$ as the table below shows.

\begin{tabular}{|c|c|c|c|}
\hline$h$ & $k$ & $\left\|w-w^{h, 1 / k}\right\|_{L^{\infty}(0,1)}$ & $\left\|w-w^{h, 1 / k}\right\|_{L^{\infty}(0,1)} /\left(h^{2}+k^{2}\right)$ \\
\hline 0.200 & 0.025000 & 0.002512 & 0.0618 \\
0.100 & 0.012500 & 0.000519 & 0.0511 \\
0.050 & 0.006250 & 0.000118 & 0.0465 \\
0.025 & 0.003125 & 0.000029 & 0.0451 \\
\hline
\end{tabular}

Since the errors divide by roughly four while the mesh parameter $h$ and time step length $k$ divide by two we observe an order two rate of convergence. This is furthur confirmed by the fact that the last column is tending to a constant.

We do note that nonphysical negative strains were observed in these computations when $\Delta t$ was not sufficiently small.

\section{Space-Time Finite Element Method:}

We describe yet another space-time finite element method, now using piecewise linear functions for both the spatial and temporal discretizations. The time discretization is called the continuous Galerkin method (see [FS] or [FJ]). It has the desirable feature of keeping the strain positive and thus modeling the behavior of the true solution. We are also able to obtain a discrete energy inequality that resembles (9). We did not obtain the other energy estimates which involved differentiating the defining equations. To compensate for this we add several new assumptions:

(H5) There exists a positive constant $\Lambda(\eta)$ such that

$$
\left|\phi^{\prime \prime}(y)\right| \leq \Lambda(\eta)<\infty \text { and }\left|\sigma_{y}(y, z)\right| \leq \Lambda(\eta)|z|
$$

for $\eta<y<\infty$ and $-\infty<z<\infty$ where we recall the splitting (5).

Note that our example constitutive formula (16)-(17) does satisfy (H5).

We use the same grid as was defined in the previous section. Denote $\mathcal{Q}_{q}=(0,1) \times J_{q}$. Let $C_{k}$ be a set of continuous piecewise linear functions and let $D_{k}$ be a set of piecewise constant functions, not necessarily continuous, defined on the partition in time. Let $\mathcal{C}_{h k}=V^{h} \otimes C_{k}$ and $\mathcal{D}_{h k}=V^{h} \otimes D_{k}$. 
We are now in a position to define our numerical scheme. Find $\left(w^{h k}, v^{h k}\right) \in \mathcal{C}_{h k} \times \mathcal{C}_{h k}$ such that

$$
\begin{gathered}
\left(w_{t}^{h k}-v^{h k}, \chi\right)_{\mathcal{Q}}=0 \quad \forall \chi \in \mathcal{D}_{h k} \\
\left(v_{t}^{h k}, \zeta\right)_{\mathcal{Q}}+\left(n\left(w_{s}^{h k}, w_{s t}^{h k}\right), \zeta_{s}\right)_{\mathcal{Q}}=\left(n_{1}, \zeta(1, \cdot)\right)_{J}+\left(n_{0}, \zeta(0, \cdot)\right)_{J}+(f, \zeta)_{\mathcal{Q}} \quad \forall \zeta \in \mathcal{D}_{h k}
\end{gathered}
$$

where $w^{h k}(\cdot, 0) \cong w^{0}$ and $v^{h k}(\cdot, 0) \cong v^{0}$ are given. For any domain $A$ we use the inner product

$$
(\eta, \xi)_{A}=\int_{A} \eta \xi d A \text { giving }\|\eta\|_{A}=\left(\int_{A} \eta^{2} d A\right)^{1 / 2} .
$$

The problem can also be defined in a slab-by-slab manner by the equations

$$
\left(w_{t}^{h k}-v^{h k}, \chi\right)_{\mathcal{Q}_{q}}=0 \quad \forall \chi \in V^{h} \otimes P_{0}\left(J_{q}\right)
$$

and

$$
\left(v_{t}^{h k}, \zeta\right)_{\mathcal{Q}_{q}}+\left(n\left(w_{s}^{h k}, w_{s t}^{h k}\right), \zeta_{s}\right)_{\mathcal{Q}_{q}}=\left(n_{1}, \zeta(1, \cdot)\right)_{J_{q}}+\left(n_{0}, \zeta(0, \cdot)\right)_{J_{q}}+(f, \zeta)_{\mathcal{Q}_{q}} \quad \forall \zeta \in V^{h} \otimes P_{0}\left(J_{q}\right) .
$$

The discretization in time can be rewritten as a finite difference scheme. Let $w^{h k, q}=w^{h k}(\cdot, t)$ and define $v^{h k, q}$ similarly. Assuming that $w^{h k, q-1}$ and $w^{h k, q}$ are known, the approximation is found on the next step by solving the following nonlinear system for $w^{h k, q+1}$

$$
\begin{aligned}
\left(w^{h k, q+1}-2 w^{h k, q}+w^{h k, q-1}, \chi\right)+ & \frac{k^{2}}{2}\left(\frac{N\left(w_{s}^{h k, q+1}, \partial_{t} w_{s}^{h k, q+1 / 2}\right)-N\left(w_{s}^{h k, q}, \partial_{t} w_{s}^{h k, q+1 / 2}\right)}{w_{s}^{h k, q+1}-w_{s}^{h k, q}}\right. \\
+ & \left.\frac{N\left(w_{s}^{h k, q}, \partial_{t} w_{s}^{h k, q-1 / 2}\right)-N\left(w_{s}^{h k, q-1}, \partial_{t} w_{s}^{h k, q-1 / 2}\right)}{w_{s}^{h k, q}-w_{s}^{h k, q-1}}, \chi_{s}\right) \\
& =k^{2}\left(\bar{n}_{1} \chi(1)+\bar{n}_{0} \chi(0)+(\bar{f}, \chi)\right)
\end{aligned}
$$

for all $\chi \in V_{h}$ where

$$
\begin{gathered}
\bar{n}_{i}=\frac{1}{k} \int_{J_{q}} n_{i} d t \text { for } i=0,1, \quad \bar{f}=\frac{1}{k} \int_{J_{q}} f d t \\
\partial_{t} w_{s}^{h k, q+1 / 2}=\frac{1}{k}\left(w_{s}^{h k, q+1}-w_{s}^{h k, q}\right), \quad \partial_{t} w_{s}^{h k, q-1 / 2}=\frac{1}{k}\left(w_{s}^{h k, q}-w_{s}^{h k, q-1}\right)
\end{gathered}
$$

and

$$
N(y, z)=\int_{0}^{y} n(\tilde{y}, z) d \tilde{y} .
$$

Note that we are assuming that the spatial integrals are done exactly. In practice one would use either a fixed point or Newton iteration to solve the nonlinear system on each time step.

\section{Discrete energy bounds:}


We will develop a discrete version of the first energy inequality in this context. We first note some simple inequalities that will be useful. Let

$$
A u=\int_{0}^{1} u d s
$$

be an average of $u$ so

$$
|u(s)-A u| \leq\left\|u_{s}\right\|
$$

and

$$
\|A u\|_{J_{q}} \leq\|u\|_{Q_{q}}
$$

Defining the discrete kinetic energy

$$
K_{q}^{h k}=\frac{1}{2} \int_{0}^{1}\left(v^{h k, q}\right)^{2} d s
$$

we have, after taking $\chi=w_{t}^{h k}$ in (37),

$$
\left\|w_{t}^{h k}\right\|_{Q_{q}} \leq\left\|v^{h k}\right\|_{Q_{q}}
$$

and, since $v^{h k}$ is linear in $t$,

$$
\left\|v^{h k}\right\|_{Q_{q}} \leq 2 k \max \left\{K_{q-1}^{h k}, K_{q}^{h k}\right\} .
$$

Let $\chi=v_{t}^{h k}$ in (37) and $\zeta=w_{t}^{h k}$ in (38). Then

$$
E_{q}^{h k}+\int_{J_{q}} \int_{0}^{1} \sigma\left(w_{s}^{h k}, w_{s t}^{h k}\right) w_{s t}^{h k} d s d t=E_{q-1}^{h k}+\left(n_{1}, w_{t}^{h k}(1, \cdot)\right)_{J_{q}}-\left(n_{0}, w_{t}^{h k}(0, \cdot)\right)_{J_{q}}+\left(f, w_{t}^{h k}\right)_{Q_{q}}
$$

where

$$
E_{q}^{h k}=K_{q}^{h k}+\int_{0}^{1} \phi\left(w_{s}^{h k, q}\right) d s .
$$

We now estimate the boundary terms,

$$
\begin{aligned}
\left(n_{1}, w_{t}^{h k}(1, \cdot)\right)_{J_{q}} & =\left(n_{1},(I-A) w_{t}^{h k}(1, \cdot)\right)_{J_{q}}+\left(n_{1}, A w_{t}^{h k}(1, \cdot)\right)_{J_{q}} \\
& \leq C_{\epsilon}\left\|n_{1}\right\|_{J_{q}}^{2}+\frac{\epsilon}{4}\left\|w_{s t}^{h k}\right\|_{Q_{q}}^{2}+2 k \max \left\{K_{q-1}^{h k}, K_{q}^{h k}\right\}
\end{aligned}
$$

where we used (39), (40), (41), and (42) as well as Young's inequality

$$
|a b| \leq \epsilon a^{2}+C_{\epsilon} b^{2}
$$

for $\epsilon>0$. A similar inequality can be found for the $n_{0}$-term. Using the boundary term estimate and applying Young's inequality to the $f$-term gives,

$E_{q}^{h k}+\int_{J_{q}} \int_{0}^{1} \sigma\left(w_{s}^{h k}, w_{s t}^{h k}\right) w_{s t}^{h k} d s d t \leq E_{q-1}^{h k}+\epsilon\left\|w_{s t}^{h k}\right\|_{Q_{q}}^{2}+C_{\epsilon}\left(\left\|n_{0}\right\|_{J_{q}}^{2}+\left\|n_{1}\right\|_{J_{q}}^{2}+\|f\|_{Q_{q}}^{2}\right)+4 k \max \left\{K_{q-1}^{h k}, K_{q}^{h k}\right\}$. 
Using hypothesis (H1), choosing $\epsilon=m / 2$, and noting that

$$
\max \left\{K_{q-1}^{h k}, K_{q}^{h k}\right\} \leq E_{q-1}^{h k}+E_{q}^{h k}
$$

gives

$$
\begin{aligned}
E_{q}^{h k}+\frac{1}{2} \int_{0}^{t_{q}} \int_{0}^{1} \sigma\left(w_{s}^{h k}, w_{s t}^{h k}\right) w_{s t}^{h k} d s d t \leq & \frac{1+4 k}{1-4 k}\left(E_{q-1}^{h k}+\frac{1}{2} \int_{0}^{t_{q-1}} \int_{0}^{1} \sigma\left(w_{s}^{h k}, w_{s t}^{h k}\right) w_{s t}^{h k} d s d t\right) \\
& +C\left(\left\|n_{0}\right\|_{J_{q}}^{2}+\left\|n_{1}\right\|_{J_{q}}^{2}+\|f\|_{Q_{q}}^{2}\right)
\end{aligned}
$$

if $k$ is sufficiently small. Iterating this estimation gives the discrete energy inequality

$$
E_{q}^{h k}+\frac{1}{2} \int_{0}^{t_{q}} \int_{0}^{1} \sigma\left(w_{s}^{h k}, w_{s t}^{h k}\right) w_{s t}^{h k} d s d t \leq C_{t_{q}}\left(E_{0}^{h k}+\int_{0}^{t_{q}} n_{0}^{2} d t+\int_{0}^{t_{q}} n_{1}^{2} d t+\int_{0}^{t_{q}}\|f\|^{2} d t .\right.
$$

\section{Total compression for the numerical Method}

In this section we show that the approximation will imitate the true solution in that it never suffers total compression. A mild restriction will be placed on the time step and spatial refinement to achieve this. Just to simplify the presentation of the argument, we set $n_{1}=0$ and $f=0$.

¿From the energy inequality (43) there exists a constant $C$ such that

$$
\int_{J_{q}} \int_{I_{p}}\left(w_{s t}^{h k}\right)^{2} d s d t \leq C
$$

Since $w_{s t}^{h k}$ is a constant on $J_{q} \times I_{p}$ this becomes

$$
\left.\left|w_{s t}^{h k}\right|\right|_{I_{p} \times J_{q}} \leq \frac{C}{\sqrt{k h}}
$$

We will now show that $w_{s}^{h k}$ cannot jump from above $y_{*}$ to below $y_{*} / 2$ in one time step provided

$$
\sqrt{\frac{k}{h}} \leq \frac{y_{*}}{4 C}
$$

¿From Taylor and (44) we have

$$
w_{s}^{h k, q}=w_{s}^{h k, q-1}+\left.k w_{s t}^{h k}\right|_{I_{p} \times J_{q}} \geq y_{*}-C \sqrt{\frac{k}{h}} \geq \frac{y_{*}}{2}
$$

Suppose $y_{*} / 4 \leq w_{s}^{h k}\left(\cdot, t_{q}\right)<y_{*}$ on $I_{p}$ (Using the preceeding argument we can show that the strain cannot jump from above $y_{*} / 2$ to below $y_{*} / 4$ on one step). If it increases above $y_{*}$ then total 
compression did not occur. If it stays below $y_{*}$ then we must address the possibility of $w_{s}^{h k} \rightarrow 0$. Recall that the function $\chi_{p} \in V^{h} \otimes P_{0}\left(J_{q}\right)$ from (21). Substituting $\chi_{p}$ into (38) for $\zeta$, we have

$$
\left(v_{t}^{h k}, \chi_{p}\right)_{Q_{q+1}}=\int_{J_{q+1}} \int_{I_{p}} n\left(w_{s}^{h k}, w_{s t}^{h k}\right) d s d t
$$

and, using hypothesis (H2), we now have

$$
\left(v_{t}^{h k}, \chi_{p}\right)_{Q_{q+1}} \leq A k h+M \int_{J_{q+1}} \int_{I_{p}} \psi\left(w_{s}^{h k}\right) d s d t-\int_{J_{q+1}} \int_{I_{p}} \psi^{\prime}\left(w_{s}^{h k}\right) w_{s t}^{h k} d s d t
$$

Simplifying, we have on $I_{p}$ that

$$
\psi\left(w_{s}^{h k, q+1}\right) \leq \psi\left(w_{s}^{h k, q}\right)+M \int_{J_{q+1}} \psi\left(w_{s}^{h k}\right) d t+\frac{1}{h}\left(\int_{0}^{1} v^{h k, q+1} \chi_{p} d s-\int_{0}^{1} v^{h k, q} \chi_{p} d s\right)+A k .
$$

Since

$$
\int_{J_{q+1}} \psi\left(w_{s}^{h k}\right) d t \leq k\left(\psi\left(w_{s}^{h k, q+1}\right)+\psi\left(w_{s}^{h k, q+1}\right)\right)
$$

we have on $I_{p}$ (for $1-M k \geq 1 / 2$ )

$$
\psi\left(w_{s}^{h k, q+1}\right) \leq \frac{1+M k}{1-M k} \psi\left(w_{s}^{h k, q}\right)+\frac{2}{h}\left(\int_{0}^{1} v^{h k, q+1} \chi_{p} d s-\int_{0}^{1} v^{h k, q} \chi_{p} d s\right)+2 A k .
$$

Iterating this inequality gives for any integer $r>q$ and since $t_{r}-t_{q}<T$ that

$$
\begin{aligned}
\psi\left(w_{s}^{h k, r}\right) & \leq C_{T}\left(\psi\left(w_{s}^{h k, q}\right)+\frac{2}{h}\left(\int_{0}^{1} v^{h k, r} \chi_{p} d s-\int_{0}^{1} v^{h k, q} \chi_{p} d s\right)+2 A T\right. \\
& \left.\leq C_{T}\left(\psi\left(\frac{y_{*}}{4}\right)+2 K_{r}^{h k}+K_{q}^{h k}\right)+2 A T\right) .
\end{aligned}
$$

Since the right side of the equation is bounded there must be a constant $\bar{y}_{*}^{F D}$ such that

$$
w_{s}^{h k} \geq \bar{y}_{*}^{F D}>0 \text {. }
$$

\section{Existence and uniqueness for the space time method:}

In this section we use the strategies given in section 3 to show that the numerical scheme (37)-(38) has a unique solution under the constraint that $k \leq \delta h$ for some sufficiently small constant $\delta$.

For existence we use the version of the Brouwer fixed-point theorem which we employed earlier. Let $H=V^{h} \otimes P_{0}\left(I_{n}\right)$, with inner product $(\cdot, \cdot)_{Q_{q}}$, and norm $\|\cdot\|_{Q_{q}}$ with $g=w_{t}^{h k}$. Then since

$$
w^{h k}(\cdot, t)=w^{h k, q-1}+\int_{t_{q-1}}^{t} g(\cdot, s) d s
$$


we can reconstruct $w^{h k}$ from $g$. Define

$$
(\Psi(g), \chi)_{Q_{q}}=\left(v_{t}^{h k}, \chi\right)_{Q_{q}}+\left(n\left(w_{s}^{h k}, g_{s}\right), \chi_{s}\right)_{Q_{q}}-\left(n_{1}, \chi(1, \cdot)\right)_{J_{q}}-\left(n_{0}, \chi(0, \cdot)\right)_{J_{q}}-(f, \chi)_{Q_{q}}
$$

where $v^{h k}$ is defined from $g$ and $v^{h k, q-1}$ through (37). Taking $\chi=g$ and arguing as we did in the energy inequality (to prove (43)), we obtain

$$
(\Psi(g), g)_{Q_{q}} \geq(1-C k) E_{q}^{h k}-(1+C k) E_{q-1}^{h k}-C\left(\left\|n_{1}\right\|_{J_{q}}^{2}+\left\|n_{0}\right\|_{J_{q}}^{2}+\|f\|_{Q_{q}}^{2}\right)+C\left\|g_{s}\right\|_{Q_{q}}^{2} .
$$

Using the Poincaré inequality, there is a positive constant $C$ such that

$$
\left\|g_{s}\right\| \geq C\left(\|g\|-\left|\int_{0}^{1} g d s\right|\right)
$$

so

$$
\left\|g_{s}\right\|_{\mathcal{Q}_{q}} \geq C\left(\|g\|_{\mathcal{Q}_{q}}-C k\left(E_{q}^{h k}+E_{q-1}^{h k}\right)\right)
$$

Thus for sufficiently small $k$ we have

$$
(\Psi(g), g) \geq C\|g\|_{\mathcal{Q}_{q}}-C\left(E_{q-1}^{h k}+\left\|n_{1}\right\|_{J_{q}}+\left\|n_{0}\right\|_{J_{q}}+\|f\|_{Q_{q}}\right)
$$

We conclude that one can force

$$
(\Psi(g), g)_{Q_{q}}>0
$$

by choosing $\mu=\|g\|_{Q_{q}}$ large enough so the Brouwer theorem asserts the existence of a solution to (37) $-(38)$.

We now show there is only one solution. Let

$$
e^{v}=v^{h k}-\bar{v}^{h k} \quad \text { and } \quad e^{w}=w^{h k}-\bar{w}^{h k}
$$

where $e^{v}\left(\cdot, t_{q-1}\right)=e^{w}\left(\cdot, t_{q-1}\right)=0$ and $\left(w^{h k}, v^{h k}\right)$ and $\left(\bar{w}^{h k}, \bar{v}^{h k}\right)$ are solution pairs of (37)-(38). Then

$$
\left(\left(e^{v}-e_{t}^{w}\right), \chi\right)_{Q_{q}}=0 \quad \forall \chi \in V^{h} \otimes P_{0}\left(J_{q}\right)
$$

and

$$
\left(e_{t}^{v}, \zeta\right)_{Q_{q}}+\left(n\left(w_{s}^{h k}, w_{s t}^{h k}\right)-n\left(\bar{w}_{s}^{h k}, \bar{w}_{s t}^{h k}\right), \zeta_{s}\right)_{Q_{q}}=0 \quad \forall \zeta \in V^{h} \otimes P_{0}\left(J_{q}\right) .
$$

Separating the nonlinear term into $\phi^{\prime}$ and $\sigma$ components, adding and subtracting the term $\sigma\left(\bar{w}_{s}^{h k}, w_{s t}^{h k}\right)$, and applying the mean value theorem the second equation becomes

$$
\left(e_{t}^{v}, \zeta\right)_{Q_{q}}+\left(\phi^{\prime \prime}(\xi) e_{s}^{w}, \zeta_{s}\right)_{Q_{q}}+\left(\sigma_{y}\left(\gamma, w_{s}^{h k}\right) e_{s}^{w}, \zeta_{s}\right)_{Q_{q}}+\left(\sigma_{z}\left(\bar{w}_{s}^{h k}, \alpha\right) e_{s t}^{w}, \zeta_{s}\right)_{Q_{q}}=0 .
$$

Let $\chi=e_{t}^{v}$ and $\zeta=e_{t}^{w}$. Using (H5) we obtain

$$
\begin{aligned}
\left\|e^{v}\left(\cdot, t_{q}\right)\right\|^{2}+m\left\|e_{s t}^{w}\right\|_{Q_{q}}^{2} & \leq C\left(\left(\left|\phi^{\prime \prime}\right|+\left|\sigma_{y}\right|\right)\left|e_{s}^{w}\right|,\left|e_{s t}^{w}\right|\right)_{Q_{q}} \\
& \leq C\left(\left(\left|e_{s}^{w}\right|,\left|e_{s t}^{w}\right|\right)_{Q_{q}} \mid+\left(\left|w_{s t}^{h k}\right|\left|e_{s}^{w}\right|,\left|e_{s t}^{w}\right|\right)_{Q_{q}}\right) \\
& =C\left(I_{1}+I_{2}\right) .
\end{aligned}
$$


Hence

$$
I_{2} \leq\left\|e_{s}^{w}\right\|_{L^{\infty}\left(Q_{q}\right)}\left\|w_{s t}^{h k}\right\|_{Q_{q}}\left\|e_{s t}^{w}\right\|_{Q_{q}} .
$$

But the second factor is bounded from (43)

$$
\left|e_{s}^{w}(\cdot, t)\right|=\left|\int_{t_{q-1}}^{t} e_{s t}^{w}(\cdot, \tau) d \tau\right| \leq k^{1 / 2}\left\|e_{s t}^{w}\right\|_{J_{q}}
$$

and from an inverse inequality,

$$
\|\chi\|_{L^{\infty}\left(I_{p}\right)} \leq C h^{-1 / 2}\|\chi\|_{L^{2}\left(I_{p}\right)}
$$

(see $[\mathrm{C}]$ ) we have

$$
I_{2} \leq C\left(\frac{k}{h}\right)^{1 / 2}\left\|e_{s t}^{w}\right\|_{Q_{q}}^{2}
$$

Thus

$$
\begin{aligned}
\left\|e^{v}\left(\cdot, t_{q}\right)\right\|^{2}+m\left\|e_{s t}^{w}\right\|_{Q_{q}}^{2} & \leq C_{\epsilon}\left\|e_{s}^{w}\right\|_{Q_{q}}^{2}+\left(\epsilon+C\left(\frac{k}{h}\right)^{1 / 2}\right)\left\|e_{s t}^{w}\right\|_{Q_{q}}^{2} \\
& \leq\left(C_{\epsilon} k+\epsilon+C\left(\frac{k}{h}\right)^{1 / 2}\right)\left\|e_{s t}^{w}\right\|_{Q_{q}}^{2} .
\end{aligned}
$$

Choosing $\epsilon$ and $\delta$ sufficiently small and then $h$ sufficiently small (so $k$ is small) we have

$$
\left\|e^{v}\left(\cdot, t_{q}\right)\right\|^{2} \leq 0
$$

It follows that $e^{v} \equiv 0$ on $Q_{q}$ since $e^{v}\left(\cdot, t_{q-1}\right)=0$. ¿From the projection equation between $e^{v}$ and $e_{t}^{w}$ we have that $e_{t}^{w} \equiv 0$. Since $e^{w}\left(\cdot, t_{q-1}\right)=0$ we have $e^{w} \equiv 0$ proving there is a unique solution to (37)-(38).

We summarize our results from this section in the following theorem:

Theorem 4: Assume $n$ satisfies hypotheses (H0)-(H3), (H5) and the data is smooth, (A0). Then there exist positive constants $\delta$ and $h_{0}$ such that for all $0<h \leq h_{0}$ and $0<k \leq \delta h$ the problem (35)-(36) has a unique solution pair $\left(w^{h k}, v^{h k}\right)$ which satisfies the energy bound (43) and has a lower bound on the strain (46).

\section{References}

[AS] S.S. Antman and T.I. Seidman, Quasilinear hyperbolic-parabolic equations of one-dimensional viscoelasticity, J. Diff. Eqns., 124 (1996), 132-185. 
[C] P. Ciarlet, The Finite Element Method for Elliptic Problems, North Holland, Amsterdam (1978).

[FJ] D.A. French and S. Jensen, Long time behaviour of arbitrary order continuous time Galerkin schemes for some one-dimensional phase transition problems, IMA J. Num. Anal., 14 (1994), 421-442.

[FS] D.A. French and J.W. Schaeffer, Continuous finite element methods which preserve energy properties for nonlinear problems, Appl. Math. Comp., 39 (1990), 271-295.

[GR] V. Girault and P.A. Raviart, Finite Element Methods for the Navier-Stokes Equations, Springer-Verlag (1986). 